\title{
EFICACIA DE HALOXIFOP-R-METIL CON DISTINTOS COADYUVANTES
}

\author{
Sanchez, P. ${ }^{1} ;$ LutZ, A. L. ${ }^{1} ;$ Magliano, M. F. ${ }^{1 ;}$ Menapace, P. ${ }^{2}$ \& Scotta, R. R. ${ }^{1}$
}

\section{RESUMEN}

En Argentina se han confirmado trece biotipos de malezas resistentes a glifosato, ocho son gramíneas y para su control se recomiendan herbicidas como haloxifop-R-metil con el agregado de aceite mineral. En el mercado se ofrecen otros aditivos para sustituir el aceite mineral que tendrían igual o mayor efectividad y menores costos. El objetivo de este trabajo fue evaluar la eficacia de haloxifop-R-metil con diferentes coadyuvantes (aceite mineral, nonilfenol etoxilado, ésteres metílicos de aceites vegetales, alcohol lineal etoxilado y alcohol oxo polioxietilenado) sobre avena 40 días después de la siembra. Se determinó la eficacia mediante la evaluación de la senescencia y peso seco de las plantas. La mejor acción herbicida de haloxifop-R-metil fue con el agregado de aceite mineral.

Palabras clave: aditivos, malezas, graminicidas.

\begin{abstract}
Efficacy of haloxifop-r-methyl with different coadyuvants.

In Argentina, thirteen biotypes of glyphosate-resistant weeds have been confirmed, eight of these are grasses. To control grassy weeds herbicides such as haloxifop-R-methyl with the addition of mineral oil is recommended. Other additives to replace the mineral oil are currently offered in the market with equal or greater efficacy and lower costs. The aim of this work was to evaluate the efficacy of haloxifop-R-methyl with different adjuvants (mineral oils, ethoxylated nonylphenol, methyl
\end{abstract}

1.- Cátedra de Sanidad Vegetal. Facultad de Ciencias Agrarias (UNL). (3080) Esperanza, provincia de Santa Fe. Tel: 03496-426400 int.366 Email: psanchez@ffca.unl.edu.ar

2.- Departamento de Protección Vegetal. INTA Reconquista (3560) Ruta Nac. Nº11 Km 773.

Tel.: 03482-420117 int. 117.

Manuscrito recibido el 25 de octubre de 2018 y aceptado para su publicación el 11 de febrero de 2019.

Sanchez, P; Lutz, A. L.; Magliano, M. F.; Menapace, P.; Scotta, R. R. Eficacia de Haloxifop-r-metil con distintos coadyuvantes. FAVE - Ciencias Agrarias 17 (2): 7-21. CC BY-NC-SA 4.0 (c)(i)()(?) 
esters of vegetable oil, ethoxylated linear alcohol and oxo polyoxyethylenated alcohol), applied in oats 40 days after sowing. Efficacy was determined by senescence and dry weight of the plants. The best herbicide action of haloxifop-R-methyl was with the use of mineral oil as an additive.

Key words: additives, weeds, graminicides.

\section{INTRODUCCIÓN}

La adopción de la siembra directa y el uso de cultivos transgénicos permitieron la incorporación de nuevas áreas productivas $\mathrm{y}$ un incremento de los rendimientos en los principales cultivos $(17,23)$. Esto también derivó en un incremento de aplicaciones de glifosato y de malezas tolerantes y resistentes a este $(6,16,19)$. En Argentina, se han confirmado trece biotipos de malezas resistentes a glifosato, de las cuales ocho son gramíneas (22). Entre las principales malezas gramíneas se encuentran, Sorghum halepense, Eleusine indica, Cynodon hirsutus, Digitaria insularis, Lolium sp y Avena fatua $(18,20,24,25,26,27)$. Se dispone de herbicidas registrados para el control de dichas malezas, que actúan inhibiendo la enzima acetil-CoA carboxilasa, enzima plastídica que cataliza el primer paso en la biosíntesis de ácidos grasos, componentes esenciales para la producción de lípidos, fundamentales para la integridad de las membranas celulares y el crecimiento de la planta (8). Para la aplicación de estos herbicidas se recomienda el agregado de aditivos como los aceites agrícolas en dosis entre 0,5 al $1 \%$ del caldo de aplicación para aumentar la permanencia del producto en la hoja y así favorecer la penetración $(2,4)$.

En el mercado de fitosanitarios hay disponibles otros aditivos a base de nonilfenol etoxilado, alcohol líneal etoxilado y orga- nosiliconados, entre otros, con acción humectante, adherente, penetrante y antideriva que podrían reemplazar el uso de aceites agrícolas, con resultados similares, utilizando menor dosis por hectárea, favoreciendo la logística y reduciendo costos (12).

El objetivo de este trabajo fue evaluar la efectividad de haloxifop-R-metil con distintos coadyuvantes en el control de avena.

\section{MATERIALES Y MÉTODOS}

El trabajo se realizó en el campo experimental de la Facultad de Ciencias Agrarias de la Universidad Nacional del Litoral (Latitud: $31^{\circ} 44^{\prime} 20,94$ " N, Longitud: $60^{\circ} 94^{\prime} 06,84$ " O). En 2014, se evaluó el efecto de haloxifop-R-metil solo y con distintos coadyuvantes sobre plantas de avena, en dos fechas de siembra (12/08 y 16/10). Se sembraron 16 macetas de $0,5 \mathrm{~L}$ por tratamiento (Tabla 1 ), con 3 semillas de Avena sativa (variedad U15) en cada una, en una mezcla de tierra y perlita. Estas fueron regadas y se mantuvieron a capacidad de campo. Se realizó raleo de las plantas dejando una por maceta.

Las aplicaciones se realizaron a los 40 días de la siembra, con una mochila pulverizadora de $\mathrm{CO} 2$, equipada con un botalón de $2 \mathrm{~m}$, con 4 picos distanciados a $0,5 \mathrm{~m}$ y con pastillas 8001, aplicando un caudal de 77,7 L.ha-1 a una presión de 2,5 bares. Las dosis 
del herbicida y de los coadyuvantes utilizados fueron las indicadas en el marbete de cada producto. Las condiciones meteorológicas al momento de la aplicación en cada ensayo fueron, temperatura $22,9{ }^{\circ} \mathrm{C}$ y $22,4{ }^{\circ} \mathrm{C}$, humedad relativa $72 \%$ y $79,3 \%$ y velocidad de viento 1,3 y 5,1 Km.h-1, respectivamente.

A los 12 días después de la aplicación se evaluó la senescencia mediante la escala visual de ALAM (Asociación Latinoamericana de Malezas) (1), donde el valor mínimo $(0 \%)$ indica que todas las plantas quedaron vivas y sin ningún daño, y el máximo ( 90 a $100 \%$ ), todas las plantas murieron. Posteriormente se cortaron las plantas al ras del suelo y se llevaron a estufa a $70{ }^{\circ} \mathrm{C}$ hasta peso constante, para determinar el peso seco. El diseño experimental utilizado fue de bloques completos al azar con arreglo factorial, dos fechas de siembra, siete tratamientos y cuatro repeticiones $(2 \times 7 \times 4)$. Los valores de senescencia fueron transformados mediante raíz cuadrada, los datos fueron analizados mediante ANOVA y la comparación de medias se realizó con el test LSD Fisher $(\mathrm{p} \leq 0,05)$ utilizando el programa estadístico INFOSTAT (7).

\section{RESULTADOS Y DISCUSIÓN}

El tratamiento de haloxifop-R-metil con aceite mineral (T3) fue el que mayor eficacia de control tuvo, el peso seco de las plantas de avena fue entre $76 \%$ y 45 $\%$ menor que el testigo. La mayor biomasa de avena en todos los tratamientos de la primera fecha de siembra, puede deberse a condiciones de temperatura y fotoperiodo más adecuadas, favoreciendo la producción de hojas (15).

La cutícula de la planta de avena tiene una superficie áspera que repele el agua debido a la disposición de las partículas de cera. Al momento de la aplicación, las plan-

Tabla 1. Productos y dosis utilizadas en cada tratamiento.

\begin{tabular}{|c|c|}
\hline Tratamiento & Producto y Dosis \\
\hline T1 & Testigo \\
\hline T2 & Haloxifop r-metil (CE 54\%) 0,1 L.ha ${ }^{-1}$ GALANT HL® \\
\hline T3 & $\begin{array}{l}\text { Haloxifop r-metil (CE 54\%) 0,1 L.ha-1 GALANT HL }{ }^{\circledR}+\text { Aceite mineral 0,77 } \\
\text { L/ha }^{-1} \text { NIMBUS }{ }^{\circ}\end{array}$ \\
\hline T4 & $\begin{array}{l}\text { Haloxifop r-metil (CE 54\%) 0,1 L.ha }{ }^{-1} \text { GALANT HL }{ }^{\circledR}+\text { Nonilfenol etoxilado } \\
0,07 \text { L/ha }^{-1} \text { SPEEDWET MAXION }{ }^{\circledR}\end{array}$ \\
\hline T5 & $\begin{array}{l}\text { Haloxifop r-metil (EC 54\%) 0,1 L.ha }{ }^{-1} \text { GALANT HL }{ }^{\circledR}+\text { Ésteres metílicos de } \\
\text { ácidos grasos de aceites vegetales (EC 70\%) 0,2 } \text { L/ha }^{-1} \text { RIZOSPRAY } \\
\text { EXTREMO }{ }^{\circledR}\end{array}$ \\
\hline T6 & $\begin{array}{l}\text { Haloxifop r-metil (CE 54\%) 0,1 L.ha }{ }^{-1} \text { GALANT HL }{ }^{\circledR}+\text { Alcohol lineal } \\
\text { etoxilado (SL 25\%) 0,07 L/ha }{ }^{-1} \text { SPEED XION ACTIVEGEL }{ }^{\circ}\end{array}$ \\
\hline T7 & $\begin{array}{l}\text { Haloxifop r-metil (CE 54\%) 0,1 } \mathrm{L}^{-h^{-1}} \text { GALANT HL }{ }^{\circledR}+\text { alcohol oxo } \\
\text { polioxietilenado (SL 35\%) 0,07 L/ha }{ }^{-1} \text { A35T BIO }{ }^{\circledR}\end{array}$ \\
\hline
\end{tabular}


tas estaban en inicio de encañazón Z3.0 (28), en este estadio los depósitos de cera de la cutícula foliar comienzan a ser más profundos lo que dificulta la absorción de herbicidas $(9,11)$. El aceite mineral tiene acción de disolución sobre las ceras cuticulares, lo que favorece el transporte transcuticular de los graminicidas con alto $\mathrm{K}(\mathrm{ow})$, como los del grupo "fop" (haloxifop-R-metil), además los tensioactivos de la formulación facilitan el mojado de la hoja y la retención de la gota $(5,14)$. El índice $\Delta \mathrm{T}$, considerando las condiciones meteorológicas al momento de cada aplicación, fue de 4 y 6 en el primer y segundo ensayo, respectivamente. Si bien estos valores son adecuados y están dentro de lo considerado como "banda de aplicación" (3), el uso de aceite mineral disminuiría la velocidad de secado de la gota, aumentando la permanencia del herbicida en la hoja, lo que se traduce en mayor tiempo de absorción y mayor control de la maleza $(4,13,21)$. Bajo estas condiciones, los aceites son a menudo más efectivos que los surfactantes para aumentar la actividad herbicida (13).

En los 2 ensayos, la aplicación de haloxifop-R-metil sin el agregado de otros coadyuvantes (T2) y los tratamientos T4 (haloxifop-R-metil + nonilfenol etoxilado), T6 (haloxifop-R-metil + alcohol lineal etoxilado), T7 (haloxifop-R-metil + alcohol oxo polioxietilenado), registran una eficacia similar sobre el control de las plantas de avena. Esto puede deberse a que la formulación del herbicida contiene emulsionantes y otros tensioactivos, incrementando el área de contacto, la adherencia y penetración estomática, mejorando la absorción (4). El agregado de coadyuvantes distintos al aceite mineral no lograrían mayor efecto herbicida (10). Si bien en el tratamiento T5 (haloxifop-R-metil + Ésteres metílicos de aceites vegetales), un componente es aceite vegetal metilado, la dosis de aceite usada es 5 veces menor que la dosis de aceite mineral empleada en T3. En la evaluación de la eficacia con la escala de ALAM, el mejor tratamiento fue T3 (haloxifop-R-metil + aceite mineral) con valores de daño entre el

Tabla 2: Peso seco (gramos) de plantas de avena en distintos tratamientos.

\begin{tabular}{|c|c|c|}
\hline Tratamiento & Primer ensayo & Segundo ensayo \\
\hline T1 & $2,25 \pm 0,13 \mathrm{c}$ & $0,58 \pm 0,05 \mathrm{~b}$ \\
\hline T2 & $1,78 \pm 0,13 \mathrm{~b}$ & $0,58 \pm 0,05 \mathrm{~b}$ \\
\hline T3 & $0,53 \pm 0,13 \mathrm{a}$ & $0,32 \pm 0,05 \mathrm{a}$ \\
\hline T4 & $2,03 \pm 0,13 \mathrm{bc}$ & $0,52 \pm 0,05 \mathrm{~b}$ \\
\hline T5 & $2,23 \pm 0,13 \mathrm{c}$ & $0,58 \pm 0,05 \mathrm{~b}$ \\
\hline T6 & $1,98 \pm 0,13 \mathrm{bc}$ & $0,47 \pm 0,05 \mathrm{ab}$ \\
\hline T7 & $1,86 \pm 0,13 \mathrm{~b}$ & $0,61 \pm 0,05 \mathrm{~b}$ \\
\hline
\end{tabular}

Medias con igual letras en la columna no tiene diferencias significativas $(p<0,05)$ 
$56 \%$ y $66 \%$, diferenciándose del testigo y de los demás tratamientos (Fig. 1). El tratamiento T4 (haloxifop-R-metil + nonilfenol etoxilado) se diferenció estadísticamente del testigo en los 2 ensayos, si comparamos con la evaluación de eficacia mediante el peso seco, en este tratamiento hubo una sobrestimación del control utilizando la escala ALAM.

\section{CONCLUSIÓN}

La mayor eficacia de haloxifop-R-metil se obtuvo con el uso de aceite mineral. Los demás coadyuvantes no mejoraron la acción del herbicida. Las ventajas en logística y costos por menor dosis de uso del resto de los coadyuvantes, no están acompañadas con una eficacia similar.

\section{BIBLIOGRAFÍA}

1.- ALAM. 1974. Resumen del panel sobre métodos de evaluación de control de malezas en Latinoamérica. II Congreso de ALAM, Cali, Colombia. Revista de la Asociación Latinoamericana de Malezas, 1(1): 6-12

2.- ARREGUI, M.C. Y PURICELLI, E. 2018. Mecanismos de acción de plaguicidas. 4ra ed. Rosario, Argentina. 264 p.

3.- CARRANCIO, L. Y MAZZARO, R. 2018. El Delta $\mathrm{T}(\Delta \mathrm{T})$ como indicador del ambiente meteorológico para pulverizaciones. En: https://inta.gob.ar/documentos/ el-delta-t-\%E2\%88\%86t-como-indicador-del-ambiente-meteorologico-para-pulverizaciones Acceso: 23/07/2018.

4.- CASAFE. 2017. Guía de Productos Fitosanitarios para la República Argentina. Cámara de Sanidad Agropecuaria y Fertilizantes. $18^{\circ}$ edición. Rosario Argentina, 1200 p.

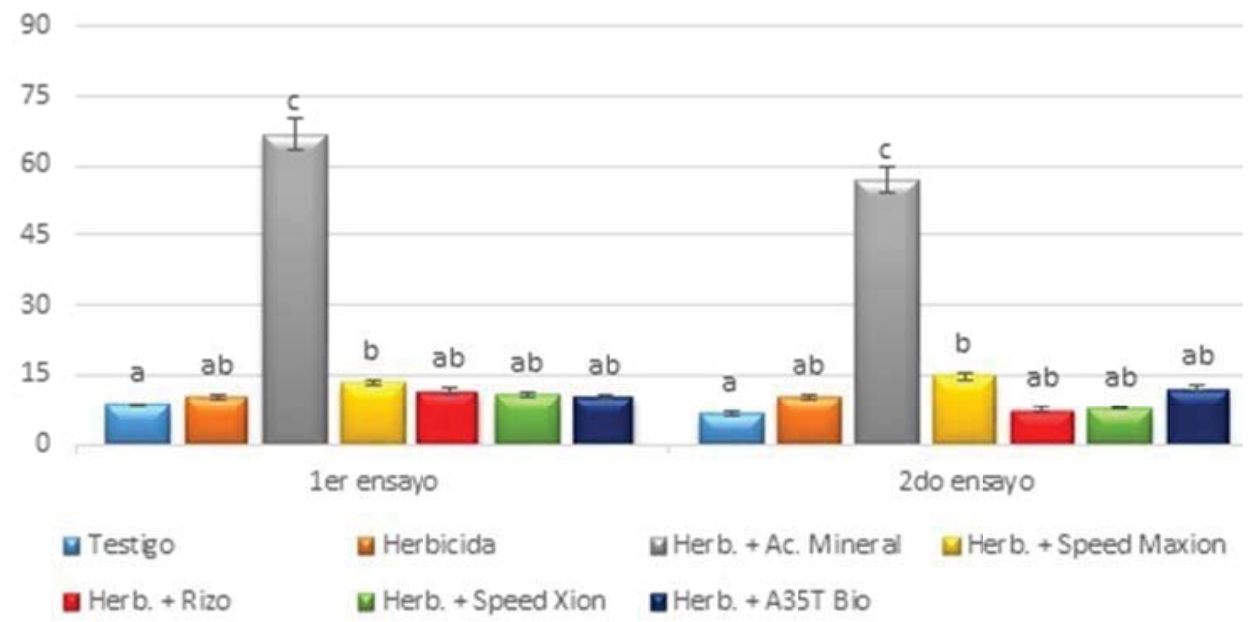

Fig. 1: Porcetnaje de control de plantas, mediante "escala visual" (ALAM, 1974). 
P. Sánchez et al.

5.- GAUVRITZ, C. Y CABANNE, F. 1992 Oils for Weed Control: Uses and Mode of Action. Pestic. Sci. 37, 147-153.

6.- DELLAFERRERA, I.; GUARISE, N. Y AMSLER, A. 2007. Relevamiento de malezas en cultivos de soja en sistema de siembra directa con glifosato del departamento San Justo (provincia de Santa Fe). Revista FAVE - Ciencias Agrarias 5 (1-2): 15-26.

7.- DI RIENZO, J.A.; CASANOVES, F.; BALZARINI, M.G.; GONZALEZ, L.; TABLADA, M. Y ROBLEDO, C.W. 2018 Grupo InfoStat, FCA, Universidad Naciona de Córdoba, Argentina.

8.- DIEZ, P. 2013. Modos de acción herbicidas. Ed. REM - AAPRESID. Rosario, Argentina. 39 p.

9.- FOOD AND AGRICULTURE ORGANIZATION OF THE UNITED NATIONS; LABRADA, R.; CASELEY, J. C. Y PARKER, C. 1996. Manejo de malezas para países en desarrollo. Food \& Agriculture Org. $403 \mathrm{p}$.

10.- FOY, CH. L. 2017. Adjuvants for Agrichemicals. Ed: CRC Press. Boca Ratón, Florida, Estados Unidos. $763 \mathrm{p}$

11.- GARCÍA TORRES, L. Y FERNÁNDEZ QUINTANILLA，C. 1991. Fundamentos sobre malas hierbas y herbicidas. Madrid: Ed. Mundi-Prensa, $348 \mathrm{p}$.

12.- IVANKOVIC, J. V.; IVANKOVIC, D. Y TRUNGELLITI, M. A. 2011. Pulverización aérea con SpeedWet NG. Conceptos para una aplicación eficiente. Revista Producción Agroindustrial del NOA 189: 72-73). [online] Disponible en: http://www. produccion.com.ar/ver_nota.php?edicion=Mar Abr2014\&numero $=189 \& i d=854$. Acceso: 24/10/201713.
13.- JALIL MALUF, E. Y ETCHEGOYEN, J. 2014. Aplicaciones agrícolas de calidad y uso de coadyuvantes de última generación. Gota Protegida. Rizobacter. p 7-83

14.- KIRKWOOD, R.C. 1999. Recent developments in our understanding of the plant cuticle as a barrier to the foliar uptake of pesticides. Pesticide Science 55(1): 69-77.

15. MACHADO, R. Y MENDOZA, F. 1987. Efecto del momento de siembra y de cosecha en la producción de forraje de Avena sativa. Pastos y Forrajes 19 (3).

16. MINISTERIO DE AGROINDUSTRIA, 2018. Estimaciones Agrícolas. En: http:// datosestimaciones.magyp.gob.ar/ Acceso: $1 / 03 / 2018$

17. MONQUERO, P.; CHRISTOFFOLETI, P.; MATAS, P. Y HEREDIA, A. 2004. Caracterização da superficie foliar e das ceras epicuticulares em Commelina benghalensis, Ipomoea grandifolia e Amaranthus hybridus. Vicosa-MG, 22: 203-210.

18. NANDULA, V.; POSTON, D.; EUBANK, T.; KOGER, C. Y REDDY, K. 2007. Differential response to glyphosate in Italian ryegrass (Lolium multiflorum) populations from Mississippi. Weed Technology. 21 (2): 477-482.

19. PAPA, J. C. 2005. Malezas: frente a un punto de vista diferente. En: http://www. e-campo.com. Acceso 10 de mayo de 2018.

20. PAPA, J. C. Y TUESCA, D. 2014. Evaluación de herbicidas para el control de Digitaria insularis (L) Mez en post-emergencia. Revista "Para mejorar la producción"-INTA EEA Oliveros 54: 159-164

21. PURICELLI, E. Y MARCH, H. 2014. Formulaciones de productos fitosanitarios para sanidad vegetal. Rosario. Ed. Rosario $1^{\mathrm{a}}$ ed. $112 \mathrm{p}$ 
22. REM. 2018. Alertas. En: http://www.aapresid.org.ar/rem/alertas/ Acceso: 4/7/2018

23. SATORRE, E. 2005. Cambios tecnológicos en la agricultura argentina actual. Ciencia hoy 15(87): 24-31.

24. USTARROZ, D. 2015. Resistencia múltiple de Sorghum halepense (sorgo de alepo) a glifosato y Haloxifop-R-metil en la provincia de Córdoba, Argentina. Informe Técnico. EEA INTA Manfredi.

25. USTARROZ, D. Y RAINERO, H. 2013 Grama carraspera (Eleusine indica) resistente a glifosato confirmada en la provincia de Córdoba Argentina. Cartilla digital Manfredi ISSN On Line 1851-7994.

26. USTARROZ, D; MAZZINI, P.; RAINERO, H. 2012. Avances en el manejo de Cynodon hirsutus resistente a glifosato. Cartilla digital Manfredi ISSN OnLine 1851-7994.

27. VILA-AIUB, M.; BALBI, M.; GUNDEL, P.; GHERSA, C. Y POWLES, S. 2007. Evolution of glyphosate-resistant johnsongrass (Sorghum halepense) in glyphosate-resistant soybean. Weed Science 55: 566571.

28. ZADOKS J.C., CHANG T.T. Y KONZAK C.F. 1974. A decimal code for the growth stages of cereals. Weed Research. 14: 415-421. 